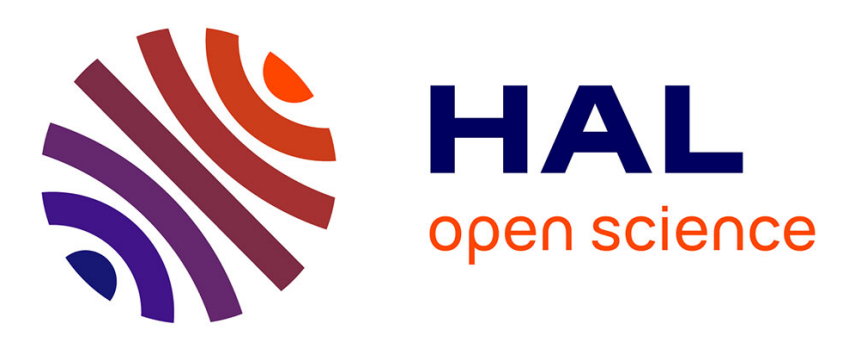

\title{
Fostering cooperation between overlay applications and underlying networks
}

Nico Schwan, Klaus Satzke, David Griffin, Bertrand Mathieu, Toufik Ahmed

\section{To cite this version:}

Nico Schwan, Klaus Satzke, David Griffin, Bertrand Mathieu, Toufik Ahmed. Fostering cooperation between overlay applications and underlying networks. Bell Labs Technical Journal, 2011, 16 (2), pp.193-198. 10.1002/bltj.20510 . hal-00958097

\section{HAL Id: hal-00958097 https://hal.science/hal-00958097}

Submitted on 11 Mar 2014

HAL is a multi-disciplinary open access archive for the deposit and dissemination of scientific research documents, whether they are published or not. The documents may come from teaching and research institutions in France or abroad, or from public or private research centers.
L'archive ouverte pluridisciplinaire HAL, est destinée au dépôt et à la diffusion de documents scientifiques de niveau recherche, publiés ou non, émanant des établissements d'enseignement et de recherche français ou étrangers, des laboratoires publics ou privés. 
Fostering Cooperation Between Overlay Applications and Underlying Networks

Nico Schwan, Klaus Satzke, David Griffin, Bertrand Mathieu, and Toufik Ahmed 


\section{Nico Schwan \\ (Contact Author)}

Alcatel-Lucent Deutschland AG

Lorenzstrasse 10

Room 008/ 02/18

70435 Stuttgart, Germany

+49 (0) 71182132310 (voice)

+49 (0) 71182132185 (fax)

Nico.Schwan@alcatel-lucent.com

Klaus Satzke

Alcatel-Lucent Deutschland AG

Lorenzstrasse 10

Room 008/ 02/20

70435 Stuttgart, Germany

+49 (0) 71182142112 (voice)

+49 (0) 71182132185 (fax)

Klaus.Satzke@alcatel-lucent.com

David Griffin

Department of Electronic and Electrical Engineering

University College London

Torrington Place

London WC1E 7JE, United Kingdom

+44 (0) 2076797606

+44 (0) 2073889325

dgriffin@ee.ucl.ac.uk

Bertrand Mathieu

Orange Labs / CORE / TPN

2, Avenue Pierre Marzin

22307 Lannion Cedex, France

+ 33296052948 (voice)

+ 33296051470 (fax)

bertrand2.mathieu@orange-ftgroup.com

Toufik Ahmed

CNRS LaBRI Lab. - University of Bordeaux, France

351, Cours de la Libération

Talence Cedex, France

+33540003501 (voice)

+33 540006669 (fax)

tad@labri.fr 
Considering the demanding nature of future high definition $(H D)$ and three dimensional (3D) video communications, application overlays will need increased awareness of underlying network capabilities. We propose a new cross-layer solution to enhance overlay/ISP interactions by providing a comprehensive, media aware, open, and standardized interface between ISPs and application overlays. We further discuss how cooperation between the layers will be improved and describe an example use case to demonstrate the benefit of such interworking. ( 2011 Alcatel-Lucent.

\section{(head 1) Introduction}

Future networked media environments will be high quality, multi-sensory, multi-viewpoint, and multistreamed and will rely on high definition (HD) and three dimensional (3D) video [1]. For Internet service providers (ISPs), however, this will place unprecedented demands [4] on the networks for high capacity, low latency, low loss and reliable communication paths. Because the traditional solution of pre-provisioning/overprovisioning of sufficient network resources everywhere is not economically viable $[1,9]$ given the huge capacities required, we propose a fundamentally different approach, via a specific interface, to improve cooperation between ISPs and application overlays so that:

- Intelligent overlay applications are optimized for end-to-end performance at a global scale, being aware of the actual capabilities of multiple underlying ISPs.

- Specialized network services such as multicast distribution or in-network caching are dynamically invoked in the underlying ISPs by the applications.

- Content, and the way it is generated, accessed, and distributed is adapted on-the-fly to what the networks are able to deliver and according to terminal capabilities.

Due to the lack of a vertical interface that allows cooperative information exchange and service invocation, today's Internet overlay applications, as well as the underlying networks, are using independent and thus often counterproductive content routing strategies. Cooperation will benefit both sides through a mutual optimization of the content delivery. The Internet Engineering Task Force (IETF) has specified the ApplicationLayer Traffic Optimization (ALTO) protocol [6] to begin to bridge this gap. In contrast to ALTO, we propose to establish a much richer interface to exchange additional information and also to invoke services. Projects like NAPA WINE [2] and SmoothIT [3] are also working to bridge the same gap, but our approach supports more sophisticated applications that are multi-participatory, multi-sourced, and interactive. 


\section{(head 1) ENVISION Interface}

Current overlay applications are, for the most part, unaware of the underlying network infrastructure since there is no established means for the exchange of cross-layer information. To overcome this limitation, overlay networks try to optimize their topology based on end-to-end measurements [5] or by reverse engineering the network, however this optimization often undermines ISP routing policies [6, 8]. Further, ISPs today are not part of the value chain of over-the-top applications, partly because there is no standardized way for them to offer value-added services to applications without significant offline pre-provisioning efforts. Our current work focuses on overcoming these limitations by facilitating cooperation between overlay applications and ISP layers via the ENVISION interface. Figure 1 highlights this interface (blue arrows) between different networks and the overlay layer. The interface enables the exchange of information and the invocation of network services offered by ISPs.

Numerous candidate network services could be offered via the interface. Some examples of those under development include the following.

1. Multicasting. Since applications may be spread over several ISPs, native intra-domain multicast mechanisms can be enhanced with techniques such as hybrid application-network layer multicast or operator-instantiated high capacity nodes in the network.

2. Caching. Employing specialized nodes provided by the ISP or third parties can save bandwidth in the network. Caching can also aid in the provisioning of catch-up services.

3. Bandwidth on demand. Edge devices may be connected to multiple access networks. Methods for provisioning capacity-on-demand over aggregated access networks are being investigated.

4. Dynamic QoS mapping. Application QoS requirements may be invoked and mapped dynamically to network capabilities, end user devices, and access networks.

5. Advertisement/text insertion. Network operators may provide added-value services that also present an opportunity for monetization.

6. Content adaptation. Heterogeneous end user devices and access network capabilities will require dynamic and distributed multimedia content adaptation techniques.

In comparison to the ALTO approach, we propose to extend the scope of the information exchanged with a rich set of data, and with the introduction of a two-way information exchange. Beyond providing applications with network topology information, network operators also may want to provide information about the access network itself (e.g., type, link capabilities, coverage), current network services status (e.g., number of 
multicast groups, content population), as well as additional metrics such as router load or bandwidth delay.

Furthermore, overlays can keep network operators apprised about the applications running within their networks and enable the optimization of data delivery by providing information on users (e.g., estimated total number of users and locations) and on content (e.g., number of different sources, bit rate, and adaptive coding).

The information exchange as well as the invocation of network services will lead to cost reductions for the network operator through more optimized content distribution. Operators will also benefit from access to application-specific information that was formerly unavailable to them, and through new revenue models by offering services to overlay applications. In this way, future network investments can be shared between the ISP and the applications that are using the network. An application, in turn, will be able to offer improved quality of service (QoS) to its users, leading to a win-win situation for all parties.

\section{(head 2) Cooperation Between Layers}

The success of emerging networked media environments is driven by services and applications that can be personalized and location-aware, and that can therefore adapt their content according to the user context and network information. The comprehensiveness and content awareness of the ENVISION interface will enable the layers to cooperate for the mutual optimization of both the overlay application and the network resources. One of the primary research challenges for this optimization is to construct an end-to-end knowledge by aggregating and harmonizing information coming from both the application and network layers, and from different network domains. At the same time, the associated management overhead introduced through this cooperation has to be kept to a minimum.

In today's network, multimedia content delivery services are centrally managed by provider-side logic with limited or no knowledge of the underlying consumer's network topology and conditions, user requirements, and the usage environment. Offline pre-generation strategies have been used to cope with dynamics and heterogeneous delivery by pre-adaptation of the most popular content. However, this solution cannot meet continuously changing user interests and the huge variation in the capabilities of both heterogeneous terminals and access networks. One goal is thus the improvement of content adaptation services by enabling dynamic, distributed, and on-the-fly content adaptation that can be coordinated through the ENVISION interface. This includes real time encoding and adaptation of content for transmission to a large number of users, processing and mixing of media sources to produce derived content, content customization and tailoring, and distribution of the derived content to the consumer. 
Another key challenge is the utilization of network services that are currently hidden from overlay applications. Multicast, for example, is not accessible to today's applications and service providers, mainly because it is not globally deployed. To overcome these limitations, overlay nodes can be classified through interactions via the ENVISION interface according to the underlying network capabilities. This allows the overlay application logic to identify nodes belonging to the same domain where a multicast address can be established for local content distribution, and thereby significantly reduce the load on the network. As an example, the Abilene [7] core network topology of 11 nodes and 14 links was assumed for the ISP. Assuming equal link metrics, a stream bandwidth of $\mathrm{S} \mathrm{Mb/s}$, a single source located at one of the Abilene nodes, and stream consumers at each of the other network nodes, in this particular example we found that a 58.9 percent reduction in total traffic could be achieved through the invocation of native multicast compared to traditional solutions, as detailed in Table $\mathbf{I}$.

(head 2) Bicycle Race Application Use Case

Application use cases were defined to highlight the advantages of the ENVISION solution for crosslayer cooperation. One example is the live coverage of a bicycle race involving a group of athletes in a potentially remote racing environment. Local spectators, professional news and media organizations, and even the athletes themselves generate video footage of the event, e.g., from mobile phones, dedicated video cameras or helmet-cams, from their current location. End users are able to follow the race on their individual terminals, selecting viewpoints and camera angles, following individual cyclists or specific events according to their individual interest.

This use case offers a good illustration of our solution for the following reasons:

1. It may span a large geographical region and different network operators. For instance, the dashed cloud in the underlay shown in Figure 2 represents the physical location of the event, where three people are filming the event (the content producers, two connected to ISP A and one to ISP B) and two content consumers are watching it.

2. The competitors and professional camera teams are constantly moving, while spectators' video sources, spread along the race track, are rather fixed.

3. End users (content consumers) can enjoy the service from various devices and access networks and may specify their restrictions/preferences as to the format of the received video, dynamically navigate across the racetrack, or define criteria for feeds of interest. 
4. It enables the insertion of additional information, e.g., advertisements or details about the event's location or environment, and can support video chat between viewers.

All of these features impact content delivery and require the exchange of information between actors in order to deliver content with the required QoS, especially since in a live event, the availability of sources can change quickly in the swarm. Typically, trusted and authenticated entities in the overlay (grey boxes) can push or pull information to or from the network via the ENVISION interface (step A-1, D-1). Non-authorized nodes may retrieve non-critical information (C-1). Nodes can request the instantiation of network services (service nodes), e.g., multicast in the ISP domain D, because many peers are watching the same source (step D-2), or the activation of caches in ISP domain A, because viewers want an action replay of a certain scene (step A-2). Some overlay nodes can themselves offer services, such as a content adaptation service (C-1), to fit end user requirements.

\section{(head 1) Conclusion}

In this paper, we have introduced the concept of a new interface between overlays and the underlying network infrastructure with the goal of fostering cooperation between those layers to optimize the operation and delivery of future media applications, and thereby improve their quality. We have highlighted potential information that can be exchanged as well as network services that can be invoked through the interface.

Future work will concentrate on refining the interface specifications, developing specific protocols and optimization and adaptation algorithms, and further evaluating the approach through a combination of simulations and testbed experiments.

\section{(head 2) Acknowledgements}

This work was supported by the ENVISION project (http://www.envision-project.org), a research project partially funded by the European Commission under its Seventh Framework Programme (contract no. 248565). The authors want to thank all partners for their valuable comments and proposals in various discussions during the ENVISION project.

\section{(head 2) References}

[1] Cisco, “Cisco Visual Networking Index: Forecast and Methodology, 2009-2014,” White Paper, June 2, 2010.

[2] European Commission, Seventh Framework Programme, "NAPA WINE," FP7-ICT Project, <http://www.napa-wine.eu/>. 
[3] European Commission, Seventh Framework Programme, “SmoothIT,” FP7-ICT Project, $<$ http://www.smoothit.org $>$.

[4] Future Media Internet Task Force, "Future Media Internet Research Challenges and the Road Ahead," FMI-TF White Paper, Apr. 2010.

[5] V. Gurbani, V. Hilt, I. Rimac, M. Tomsu, and E. Marocco, “A Survey of Research on the ApplicationLayer Traffic Optimization Problem and the Need for Layer Cooperation,” IEEE Commun. Mag., 47:8 (2009), 107-112.

[6] Internet Engineering Task Force, “Application-Layer Traffic Optimization (ALTO),” IETF Working Group, <http://datatracker.ietf.org/wg/alto/charter/>.

[7] Internet2, “Internet2 Network," <http://www.internet2.edu/pubs/200502-IS-AN.pdf>.

[8] R. Keralapura, N. Taft, C.-N. Chuah, and G. Iannaccone, "Can ISPs Take the Heat from Overlay Networks?," Proc. 3rd ACM Workshop on Hot Topics in Networks-III (ACM HotNets-III) (San Diego, CA, 2004).

[9] Velocix, “Enabling Digital Media Content Delivery: Emerging Opportunities for Network Service Providers,” White Paper, Mar. 2010, <http://www.velocix.com/VideoWeb.pdf>.

(Manuscript approved February 2011) 
NICO SCHWAN is a research engineer with the Alcatel-Lucent Bell Labs' Service Infrastructure Research Team in Stuttgart, Germany. He is also an academic at the University of Cooperative Education, Stuttgart. His current research interests are in the areas of content delivery networks, multimedia Internet overlay applications, and content-centric networking. Mr. Schwan has a B.Sc. degree in applied computer science from the University of Cooperative Education, Stuttgart.

KLAUS SATZKE is a senior researcher at Alcatel-Lucent Bell Labs in Stuttgart, Germany. He received his diploma and Ph.D. degrees in physics from Philipps University in Marburg, Germany. He spent one year on a post-doctoral fellowship at the Centre National d'Etudes des Telecommunications (CNET) research center in Paris, France, and a second year in the Advanced Sources department at BNR Europe Research Labs in Harlow, United Kingdom. He joined the Alcatel SEL Research Center in Stuttgart 19 years ago. His early responsibilities included multiple research projects in the context of prototype realization, measurement, and performance evaluations for broadband transmission systems, and he made a number of contributions to the European RACE, AQUA, and ACTS Highway research programs. For the last six years, his research focus has been in the area of network computing and rich media services over Internet overlay networks. He has had substantial experience in managing R\&D teams including teams from EU-funded research activities such as SerCHo, G-Labs, OPUCE, ENVISION, and IRMOS. Dr. Satzke is a longstanding member of the Alcatel-Lucent Technical Academy, and is currently acting as co-chair of the EU Collaboration Work Group on Virtualized Service Platforms.

DAVID GRIFFIN is a principal research associate in the Department of Electronic and Electrical Engineering, University College London. He has a B.Sc. from Loughborough University, Leicestershire, England, and a Ph.D. from UCL, both in electrical engineering. Dr. Griffin's research interests are in planning, management, and dynamic control for providing QoS in multiservice networks, P2P networking, and novel routing paradigms for the future Internet.

BERTRAND MATHIEU is a senior researcher at France Telecom, Orange Labs. He received a diploma in engineering at the Universite du Sud Toulon-Var, an Ms.C. degree from the University of Marseille, and a Ph.D. degree from the University Pierre et Marie Curie in Paris. His research 
activities are related to dynamic overlay networks, P2P networks, and information-centric networking. Dr. Mathieu has contributed to several national and European projects and has published more than 30 papers in international conferences, journals, or books. He also serves on the Technical Program Committee for several conferences, and is a senior member of the IEEE and SEE.

TOUFIK AHMED is a full professor at the Institut Polytechnique de Bordeaux (IPB) in the ENSEIRBMATMECA school of engineering. He received his B.Sc. in computer engineering with high honors from the National Institute of Computer Science (I.N.I.) Algiers, Algeria, and an M.Sc. and Ph.D. degree in computer science from the University of Versailles, France. He also obtained the HDR (Habilitation à Diriger des Recherches) degree at University of Bordeaux-1 on adaptive streaming and control of video quality of service over wired/wireless IP networks and P2P architectures. Dr. Ahmed served previously as a visiting scientist at the School of Computer Science at the University of Waterloo, and as a research fellow at the PRISM laboratory at the University of Versailles. His primary research activities concern quality of service for multimedia wired and wireless networks, end-to-end signaling protocols, P2P networks, and wireless sensor networks. 
(panel head) Panel 1. Abbreviations, Acronyms, and Terms

3D-Three dimensional

ALTO_Application-Layer Traffic Optimization

HD—High definition

IETF-Internet Engineering Task Force

ISP_-Internet service provider

NAPA WINE-Network-Aware P2P-TV Application over Wise Networks

P2P_Point-to-point

QoS-Quality of service

SmoothIT_Simple Economic Management Approaches of Overlay Traffic in Heterogeneous Internet Topologies

TV-Television 
Table I. Network service example.

\begin{tabular}{|l|c|c|c|}
\hline Distribution mode & Maximum link load & Source upload capacity & $\begin{array}{c}\text { Total load } \\
\text { (sum of link load) }\end{array}$ \\
\hline Client - server $^{\star}$ & $4 \mathrm{~S}-8 \mathrm{~S}$ & $10 \mathrm{~S}$ & $21 \mathrm{~S}-30 \mathrm{~S}$ \\
\hline P2P swarming & $>1 \mathrm{~S}$ & $1 \mathrm{~S}$ & $26.8 \mathrm{~S}$ \\
\hline Native multicast & $1 \mathrm{~S}$ & $1 \mathrm{~S}$ & $11 \mathrm{~S}$ \\
\hline
\end{tabular}

${ }^{*}$ Results depend on source node location

** Assuming equal load distribution

P2P_-Point-to-point 